Cahiers de philosophie de l'université de

\title{
L'interprétation performative du cogito cartésien
}

\author{
Elena Dragalina-Chernaya
}

\section{(2) OpenEdition}

Journals

Édition électronique

URL : https://journals.openedition.org/cpuc/727

DOI : $10.4000 /$ cpuc.727

ISSN : 2677-6529

Éditeur

Presses universitaires de Caen

\section{Édition imprimée}

Date de publication : 31 décembre 2013

Pagination : 121-139

ISBN : 978-2-84133-487-2

ISSN : 1282-6545

\section{Référence électronique}

Elena Dragalina-Chernaya, « L'interprétation performative

du cogito cartésien », Cahiers de philosophie de l'université de Caen [En ligne], 50 | 2013, mis en ligne le

13 juin 2018, consulté le 02 février 2023. URL : http://journals.openedition.org/cpuc/727 ; DOI : https://doi.org/10.4000/cpuc.727

\section{(c) (7) (8)}

Creative Commons - Attribution - Pas d'Utilisation Commerciale 4.0 International - CC BY-NC 4.0 https://creativecommons.org/licenses/by-nc/4.0/ 


\title{
L'interprétation performative du cogito cartésien ${ }^{1}$
}

Les paroles toutefois m'arrestent, et je suis presque trompé par les termes du langage ordinaire.

Méditations, AT IX-1, 25.

\begin{abstract}
T 'EGO SUM, EGO EXISTO ${ }^{2}$ dont la certitude vient de «l'infaillible innocence de l'expérience» du cogito, selon les termes de Jean-Marie Beyssade ${ }^{3}$, relève-t-il des «actes de discours» dont la valeur de vérité dépend d'une situation d'énonciation? On le sait, Jaakko Hintikka a présenté ce qu'on peut appeler «l'interprétation performative du cogito» dans une série d'articles publiés depuis $1962^{4}$. L'idée principale de cette interprétation consiste à déplacer l'attention des propositions vers les jugements. Alors
\end{abstract}

1. Dans cet article sont utilisés les résultats du projet réalisé dans le cadre du programme de recherches de 2013: Онтология негативности, de l'École des hautes études en sciences économiques, Université d'État, Moscou.

2. Meditatio secunda, in Meditationes, AT VII, 27.

3. Voir J.-M. Beyssade, La philosophie première de Descartes. Le temps et la cohérence de la métaphysique, Paris, Flammarion (Nouvelle bibliothèque scientifique), 1979, p. 253: «La force extrême du Cogito tient à la possibilité continuelle de réduire à rien la distance entre la proposition et l'expérience qu'elle se propose d'exprimer. Mais son seul statut de proposition, en lui faisant perdre l'infaillible innocence de l'expérience, ou de la connaissance intérieure, en transformant la nature en discours, et l'évidence en science, constitue sa fragilité, sinon sa faiblesse».

4. J. Hintikka, "Cogito ergo sum: inférence ou performance?», Philosophie, $\mathrm{n}^{\circ} 6,1985$, p. 21-51, trad. fr. de P. Le Quellec-Wolff de l'article «Cogito ergo sum: Inference or Performance? », Philosophical Review, vol. 71, $\mathrm{n}^{\circ}$ 1, 1962, p. 3-32; J. Hintikka, «The Cartesian Cogito, Epistemic Logic and Neuroscience: Some Surprising Interrelations ", Synthese, vol. 83, n ${ }^{\circ}$ 1, 1990, p. 133-157; J. Hintikka, "Cogito ergo sum, comme inférence et comme performance», Revue de métaphysique et de morale, $\mathrm{n}^{\circ}$ 1, 2000, p. 3-12; J. Hintikka, "Cogito, ergo quis est?», Revue internationale de philosophie, t. 50, $\mathrm{n}^{\circ}$ 195, 1996, p. 5-21. Voir à ce sujet l’article «René pense donc Cartesius existe» de J. Hintikka dans la présente revue. 
que les propositions peuvent être vraies ou fausses, les jugements, considérés comme des actes de discours, peuvent être effectués ou non. Selon l'interprétation performative:

Descartes n'infère pas le sum du cogito, mais il démontre à lui-même sa propre existence en effectuant un acte de pensée. L'expression cogito ne constitue pas une prémisse dont le sum est inféré mais un acte de pensée qui révèle (aussi longtemps qu'il dure), à Descartes, l'entité qu'il est ${ }^{5}$.

Dans l'expérience principielle du cogito, l'essentiel ne résiderait donc pas dans la liaison entre les différentes parties du jugement en termes de vérité ou de fausseté, mais plutôt dans la réalisation du jugement «je suis» comme acte de discours. Dans son interprétation, Hintikka s'appuie sur la notion d'inconsistance existentielle. Il la définit de la façon suivante: la proposition $p$ est existentiellement inconsistante pour la personne $a$ qui dit $p$ si la proposition " $p$, et $a$ existe» est contradictoire au sens habituel ${ }^{6}$.

Si $p$ est existentiellement inconsistante pour la personne $a$, non- $p$ est existentiellement autovérifiante pour $a$. Puisque la proposition « je ne suis pas et je suis» est contradictoire au sens habituel du terme, et puisque le «je» représente toujours le locuteur, la proposition «je suis» est autovérifiante pour chaque locuteur.

L'interprétation performative du cogito proposée par Hintikka a rencontré de nombreuses objections. Pour l'essentiel, les critiques consistent à mettre en évidence les difficultés rencontrées dans les deux types de logique. Dans la logique avec présupposition existentielle, d'une part, on déduit la proposition «je suis» de la proposition «j'énonce que je suis». Or, dans cette logique, «je suis» peut être déduit également de la proposition « je me promène». D’autre part, dans la logique libre (libre de toute présupposition) qui autorise à parler d'actions mentales produites par des individus qui n'existent pas réellement, la prononciation par Hamlet de l'affirmation «je suis» n'implique pas l'existence réelle de Hamlet.

Je crois que cette critique de l'approche de Hintikka, qui déplace l'interprétation du niveau des actes de discours vers le niveau des propositions portant sur ces actes, n'affecte pas l'interprétation performative du cogito,

5. J. Hintikka, «The Cartesian Cogito, Epistemic Logic and Neuroscience...», p. 133: «Descartes is not inferring sum from cogito, but demonstrating to himself his own existence by performing an act of thinking. The expression cogito does not mark a premise from which sum is inferred, but a thought-act which reveals (as long as it goes on) to Descartes the entity that he is".

6. J. Hintikka, «Cogito ergo sum: Inference or Performance?», 1962, p. 11: «We shall say that $\mathrm{p}$ is existentially inconsistent for the person referred to by a to utter if and only if the longer sentence " $\mathrm{p}$; and a exists" is inconsistent (in the ordinary sense of the word)». 
dans la mesure où celle-ci présuppose une explication formelle en termes immédiats d'actes de discours. Il me semble que le moyen naturel pour cette explication est l'appareil du calcul des actes de discours' ${ }^{7}$. La notion de but illocutoire est fondamentale dans ce genre de calcul: il s'agit de l'objectif de l'acte de discours qui lui est inhérent. En effectuant l'acte de discours à but déclaratif, par exemple, le locuteur engendre un certain état de choses dans le cas d'une performance réussie. Comme le remarquent John R. Searle et Daniel Vanderveken, le but illocutoire d'une déclaration est « de changer le monde en disant quelque chose: dans les énonciations à but déclaratif, le locuteur réalise l'état de choses représenté par le contenu propositionnel uniquement en vertu de sa performance réussie d'acte de discours» ${ }^{8}$.

Les exemples déclaratifs suivants sont bien connus: "prononcer un arrêt ", «nommer», " excommunier», "déclarer mari et femme», "bénir», etc. La performance réussie du déclaratif (par exemple l'acte de nomination ou l'acte de déclaration de guerre) garantit la concordance entre son contenu propositionnel et le monde réel. Ainsi, si je donne à mon article le titre "L'interprétation performative du cogito cartésien", il le devient ipso facto.

L'interprétation performative du cogito que je propose consiste à traiter celui-ci comme un acte déclaratif dans des mondes possibles illocutoirement impossibles. La notion de monde possible illocutoirement impossible peut être définie de la façon suivante:

Soit $v$ un monde possible, $a$ un sujet de l'acte de discours effectué dans $v$. Le monde $v$ est illocutoirement impossible si et seulement si $a \ddot{I} \mathrm{D}(v)$, où $\mathrm{D}(v)$ est un domaine du monde $v$.

Dans le cadre de la logique illocutoire, les mondes dans lesquels les sujets des actes de discours effectués n'existent pas sont impossibles. Par conséquent, «je suis» est nécessairement vrai, et la performance de cet acte de discours dans tout monde illocutoirement possible est aussi triviale que l'énonciation d'une tautologie dans un monde logiquement possible: une telle énonciation n'élimine aucune alternative, puisque cette tautologie est déjà présupposée par la construction des mondes logiquement possibles (c'est-à-dire des mondes complets et consistants). L'affirmation «je suis» n'élimine aucune alternative dans la sémantique des mondes illocutoirement possibles, dans

7. J. R. Searle, D. Vanderveken, Foundations of Illocutionary Logic, Cambridge, Cambridge University Press, 1985, chap. 1, «Introduction to the Theory of Speech Acts», p. 1-126.

8. J. R. Searle, D. Vanderveken, Foundations of Illocutionary Logic, chap. 2, «Basic Notions of a Calculus of Speech Acts", p. 37: «[...] to change the world by saying so: in utterances with the declarative point the speaker brings about the state of affairs represented by the propositional content solely in virtue of his successful performance of the speech act». 
la mesure où l'existence du locuteur est présupposée par la construction du modèle. Ainsi, le caractère référentiel du terme «je» est simplement postulé.

Revenons donc à Descartes. Dans la «Méditation seconde», il pose la question suivante: "[j]e suis, j'existe: cela est certain; mais combien de temps?» et il donne la réponse concrète: «[a] sçavoir, autant de temps que je pense; car peut-estre se pouroit-il faire, si je cessois de penser, que je cesserois en mesme temps d'estre ou d'exister $"$ 9.

Descartes situe donc la nécessité du cogito dans un cadre temporel et temporaire. De la même façon, dans la sémantique des mondes illocutoirement impossibles, le caractère péremptoire du cogito n'est pas une nécessité intemporelle de l'implication logique mais une contrainte déclarative effectuée dans le temps. Dans cette sémantique, «je pense» et «je suis» ne sont pas liés comme une prémisse et sa conclusion, mais comme le processus et son produit, comme l'action et son résultat.

Il est certain que, dans le discours ordinaire, il n'y a aucune raison de considérer «je suis» comme l'effet de la performance réussie du déclaratif «je pense» puisque, dans le cadre de la communication courante, on parle d'actes de discours effectués. Cependant, le doute hyperbolique doit éliminer toutes sortes de prémisses, y compris la prémisse de la communication effectuée. Comme le remarque Paul Ricœur:

Le sujet du doute ne souffre en aucun cas de l'absence d'autrui puisque, en perdant son ancrage, il laisse derrière lui les conditions de l'interlocution et du dialogue. On ne peut même pas dire qu'il est engagé dans un monologue, dans la mesure où le monologue s'extrait du dialogue qu'il présuppose en l'interrompant ${ }^{10}$.

Dans les conditions du doute radical, le cogito cartésien garantit la performance réussie du déclaratif «je pense» dans tous les mondes possibles illocutoirement impossibles. On peut avancer cependant une objection assez forte contre cette interprétation du cogito. En effet, la doctrine de Descartes sur la toute-puissance divine donne des raisons de douter de la pertinence qu'il y a à recourir à une doctrine des mondes possibles dans ce cadre. Ainsi, Jean-Christophe Bardout a déclaré à juste titre dans son article «Remarques sur l'impossibilité cartésienne des mondes possibles» que

9. «Méditation seconde», in Méditations, AT IX-1, 21. En latin: «Ego sum, ego existo; certum est. Quandiu autem? Nempe quandiu cogito; nam forte etiam fieri posset si cessarem ab omni cogitatione, ut illico totus esse desinerem ", AT VII, 27.

10. Paul Ricœur «The Crisis of the Cogito", Synthese, vol. 106, ${ }^{\circ}$ 1, 1996, p. 59: «The who of doubt in no way lacks others, since, by losing its anchoring, it leaves behind the conditions of interlocution and dialogue. One cannot even say that it is engaged in monologue, inasmuch as monologue retreats from the dialogue it presupposes by interrupting it». 
" pour penser des mondes possibles, à l'âge classique du moins, il convient précisément de n'être pas cartésien, et de chercher à s'ouvrir l'accès d'une rationalité supramondaine ${ }^{11}$.

Il est vrai que, selon Descartes, non seulement l'être réel mais aussi ses lois et ses fondements dépendent de Dieu. Descartes n'accepte pas que l'essence précède l'existence. L'inséparabilité de l'essence et de l'existence signifie l'inséparabilité des domaines de ce qui existe actuellement et de ce qui est simplement possible, et ces deux domaines sont également marqués par la liberté divine absolue. Bardout dit que, selon Descartes,

il semble donc qu'il nous soit impossible de spéculer sur ce que Dieu aurait pu faire, mais n'a pas fait. L'impossibilité, comme le possible, deviennent ainsi des concepts relatifs, et le possible ne s'entend lui-même que dans l'horizon d'une rationalité créée ${ }^{12}$.

La distinction même entre le possible et l'impossible est effectuée dans l'actualité à laquelle appartient notre rationalité créée. La négation de l'impossibilité logique, c'est-à-dire de l'inconsistance, n'est que la vérité relative limitée par ce que l'homme peut comprendre. Selon Descartes, la toute-puissance de Dieu ne consiste pas du tout dans sa capacité à choisir l'un des mondes possibles (dont la diversité est a priori en concordance avec les vérités éternelles) afin de l'actualiser. Les vérités éternelles ne limitent pas la toute-puissance divine; elles limitent notre capacité à connaître cette toute-puissance de Dieu comme l'explique Descartes dans les "Secondes réponses », " car toute impossibilité, ou, s'il m'est permis de me servir icy du mot de l'école, toute implicance consiste seulement en nostre concept ou pensée, qui ne peut conjoindre les idées qui se contrarient les unes les autres ${ }^{13}$.

À la différence de Descartes, Leibniz, le fondateur de la sémantique des mondes possibles, a nié l'idée de création des vérités éternelles. Il était convaincu que Dieu choisit librement l'individu qu'il crée et que son choix est toujours le choix du meilleur non pas parce que ce choix lui appartient, mais parce que cet individu contient en soi plus d'essence et n'est pas contradictoire avec les autres individus du monde possible auquel il appartient. Selon Leibniz, la possibilité exclut la contradiction et doit précéder l'existence; par conséquent, les vérités logiques telles que le principe de non-contradiction sont nécessairement inhérentes à chaque monde possible.

Cependant, cette approche n'est pas une prémisse obligatoire de la sémantique des mondes possibles. Afin de mettre en lumière le contenu

11. J.-C. Bardout, «Remarques sur l'impossibilité cartésienne des mondes possibles», Cahiers de philosophie de l'Université de Caen, $\mathrm{n}^{\circ}$ 42, 2006, Les mondes possibles, $\mathrm{p} .69$.

12. Ibid., p. 52.

13. AT IX-1, 119. 
informatif des lois logiques, la logique non classique présuppose que les mondes possibles logiquement impossibles (autrement dit, contradictoires ou incomplets) existent. Il est certain que l'appellation de «[m]onde tout nouveau ${ }^{14}$ dans la cosmologie de Descartes, comme Bardout l'a bien montré, a «le statut épistémique et heuristique d'une fiction méthodologique $»^{15}$. Selon moi, l'hypothèse que les mondes possibles logiquement impossibles existent ne contredit pas le doute hyperbolique cartésien, à supposer que cette hypothèse soit traitée comme un moyen heuristique. L'hypothèse de l'existence des mondes logiquement impossibles ne prétend pas sortir de la rationalité créée et ne lui ajoute aucune nouvelle loi absolue de l'extérieur; en revanche, celle-ci met en lumière le contenu informatif des lois logiques qui prétendent à l'absolu sans aucune raison valable, et, à ce titre, cette hypothèse manifeste bien leur caractère relatif ainsi que leur falsifiabilité. L'idée de monde illocutoirement impossible constitue à son tour une extension de l'idée de monde logiquement impossible, destinée à mettre en évidence la force illocutoire du déclaratif que constitue le cogito.

On connaît la critique de Georg Christoph Lichtenberg, selon qui ce n'est pas le cogito («je pense», «Ich denke») mais le cogitatur ("ça pense», «Es denkt») qui possède l'évidence suffisante ${ }^{16}$. Nietzsche a partagé cette idée avec Lichtenberg, affirmant que c'était une "habitude grammaticale» de traiter le cogito comme une substance. Depuis le début du XXe siècle, les philosophes analytiques ont largement accepté cette critique. Ernst Mach a été probablement le premier positiviste à ressusciter l'argument de Lichtenberg. Bertrand Russell croyait que l'évidence immédiate est mieux adaptée à l'expression «la couleur brune est vue» qu'à l'expression «je vois la couleur brune», et il a proposé de transformer «je pense» en "ça pense en moi». Selon Russell, «les formes grammaticales "Je pense", "Tu penses", "M. Jones pense" sont trompeuses si on les considère comme indiquant l'analyse d'une pensée singulière. Il vaudrait mieux dire "ça pense en moi", de la même façon que l'on dit "il pleut ici" ou, mieux encore, "il y a une pensée en moi" ${ }^{17}$.

14. «Permettez donc pour un peu de temps à vostre pensée de sortir hors de ce Monde, pour en venir voir un autre tout nouveau, que je feray naistre en sa presence dans les espaces imaginaires ", "Traité de la Lumière», Le monde, AT XI, 31.

15. J.-C. Bardout, «Remarques sur l'impossibilité cartésienne...», p. 54.

16. "Nous ne connaissons que l'existence de nos sensations, de nos représentations et de nos pensées. On devrait dire: ça pense, comme on dit: ça éclaire. Dire cogito est déjà trop dire sitôt qu'on le traduit par: je pense. Postuler le je est un besoin pratique», G. C. Lichtenberg, Schriften und Briefe. Band. 6, cité dans I. Haaz, Nietzsche et la métaphore cognitive, Paris, L'Harmattan, 2005, p. 141.

17. "the grammatical forms "I think", "you think", and "Mr. Jones thinks", are misleading if regarded as indicating an analysis of a single thought. It would be better to say "it thinks in 
À première vue, dans l'esprit de Lichtenberg, on pourrait dire que Descartes, dans ses "Septièmes objections", parle une langue sans sujet, tant celui qui profère le cogito est hors du monde de l'expérience, sans âme ni corps.

Dans le cadre de la réponse à l'objection: «Pourquoi faites-vous mention de l'esprit quand vous dites: lorsque je la conçois en mon esprit; n'avezvous pas même banni le corps et l'esprit ? ${ }^{18}$, Descartes remarque en effet:

Mais ici concevoir en son esprit ne signifie rien autre chose que penser; et partant il [le P. Bourdin] suppose mal que je fais mention de l'esprit en tant que considéré comme une partie de l'homme. De plus, encore que j'aie rejeté ci-devant le corps et l'esprit, avec tout le reste de mes anciennes opinions, comme des choses douteuses ou des choses que je ne concevais pas encore clairement, cela n'empêche pas que je ne les puisse reprendre par après s'il arrive que je les conçoive clairement ${ }^{19}$.

Cette reprise possible exige la performance réelle du cogito. Ainsi, selon Descartes, «nous ne connoissons pas la substance immediatement par elle-mesme, mais seulement parce qu'elle est le sujet de quelques actes ${ }^{20}$. D’autre part, «il est certain que la pensée ne peut pas estre sans une chose qui pense, et en général aucun accident ou aucun acte ne peut estre sans une substance de laquelle il soit l'acte ${ }^{21}$.

Dans le monde illocutoirement impossible de type cartésien, ego sum, ego existo n'est pas une tautologie ordinaire mais une tautologie féconde ${ }^{22}$, dont la vérité nécessaire peut être prouvée a posteriori, c'est-à-dire après la

me", like "it rains here"; or better still, "there is a thought in me», B. Russell, The Analysis of Mind, Londres, Macmillan, 1921, p. 18.

18. R. Descartes, «Septièmes objections et réponses", in Euvres philosophiques, F. Alquié (éd.), Paris, Garnier, 1963-1973, t. II, p. 981 (cité désormais Alquié, suivi du tome et de la page); pour le latin: "Cur mentis meministi, cum ais "mentem concipitur"? An non exulare 19. Ibid. jussisti corpus et mentem?», AT VII, 480 .

20. «Réponses aux troisièmes objections», in Méditations, AT IX-1, 136.

21. Ibid.

22. Ce terme a été introduit par le phénoménologue russe M. Mamardashvili, dans M. Mamardashvili, Картезианские размышления, Прогресс, Культура, 1993 (Kartezianskié razmichlenia [Méditations cartésiennes], Moscou, Kultura, 1993). François Récanati parle à son tour des contradictions pragmatiques. «Les contradictions pragmatiques sont intermédiaires entre les propositions synthétiques ordinaires fausses et les contradictions logiques. Les propositions synthétiques fausses sont contredites par les faits, et les contradictions logiques se contredisent elles-mêmes; les contradictions pragmatiques, elles, se contredisent elles-mêmes en étant contredites par les faits: c'est un fait contingent qui les rend fausses, comme les propositions synthétiques, mais elles sont à tel point indissociables du fait qui, les contredisant, les rend fausses, que d'être contredites par lui revient pour ces propositions à se contredire elles-mêmes», F. Récanati, La transparence et l'énonciation, Paris, Seuil (L’Ordre philosophique), 1979, p. 197. 
performance de l'acte de discours. Mais, puisque, ce qui est nécessaire ne l'est pourtant qu'a posteriori, la question de la validité de l'usage du terme logique «donc» dans l'énonciation du principe cartésien qui exprime une relation a priori reste ouverte.

On sait qu'il est impossible de traiter le cogito comme une preuve formellement correcte au sens d'un raisonnement syllogistique. Descartes a nié absolument la déduction de la conclusion «je suis » à partir de la prémisse «je pense» combinée à la majeure «tout ce qui pense, est ou existe». Dans ses «Réponses aux secondes objections», il écrit:

[...] quand nous apercevons que nous sommes des choses qui pensent, c'est une premiere notion qui n'est tirée d'aucun syllogisme; et lorsque quelqu'un dit: Je pense, donc je suis, ou j'existe, il ne conclut pas son existence de sa pensée comme par la force de quelque syllogisme, mais comme une chose connuë de soy; il la void par une simple inspection de l'esprit ${ }^{23}$.

Il n'en reste pas moins qu'il parle du cogito comme d'une conclusion (conclusio), ce qui signifie qu'il lui confère la portée d'une proposition conclue dont la vérité est établie non pas par un raisonnement, mais par l'expérience que nous en faisons. Cela n'exprime aucune réserve, mais lui reconnaît un statut de vérité aussi légitime que celui qu’une vérité pourrait recevoir d'un raisonnement formellement valide.

Cette interprétation de la preuve n'est pas devenue dominante dans la logique moderne: la définition standard de la preuve y est donnée en termes de conditions de vérité et, par conséquent, prouver une proposition signifie qu'il faut montrer qu'elle ne peut pas être fausse dès lors que toutes les prémisses acceptées sont vraies. La compréhension de la proposition revient à connaître les conditions de sa vérité. Du point de vue performatif, en revanche, la preuve est examinée d'une autre façon, à savoir, comme une succession d'actions correspondant aux principes fondamentaux de la réalisation compétente d'actes mentaux, ou encore comme l'explication de la façon dont la performance d'actes mentaux élémentaires rend possible la performance compétente d'actes mentaux complexes. Autrement dit, la preuve représente le passage non pas d'une proposition vraie à une autre, mais le passage d'une action justifiée à une autre, laquelle devient justifiée à son tour. De ce point de vue, une compréhension de l'énonciation réside dans la capacité à bien présenter sa preuve, c'est-à-dire ce qui permet de

23. AT IX-1, 110. En latin: "Cum autem advertimus nos esse res cogitantes, prima quxdam notio est, quæe ex nullo syllogismo concluditur; neque etiam cum qui dicit, ego cogito, ergo sum, sive existo, existentiam ex cogitatione per syllogismum deducit, sed tanquam rem per se notam simplici mentis intuitu agnoscit», AT VII, 140. 
justifier l'acte d'affirmation de cette énonciation. Puisque la performance du cogito est nécessaire afin de justifier l'affirmation sum, le principe de Descartes peut être interprété comme une preuve performative de ce genre.

Il s'ensuit dès lors que la dépendance performative de l'ego vis-à-vis de l'acte mental aggrave la question fondamentale relative au statut ontologique de l'essence de l'ego, dont l'existence est démontrée par la méthode de Descartes. Cette question est au cœur de nombreux débats. Ainsi, les débats utilisant l'appareil de la sémantique logique font appel à un principe fondamental de la philosophie analytique: il s'agit du principe de Quine, «No entity without identity» («Pas d'entité sans identité»). Dans les termes de la sémantique des mondes possibles, la réponse à la question relative au statut ontologique du «je» cartésien présuppose la spécification des critères de son identification croisée dans les mondes possibles, autrement dit dans les différents scénarios ou systèmes d'identification.

Hintikka distingue deux méthodes d'identification croisée : la méthode publique (objecto-centrée), et la méthode perspectivale (subjecto-centrée). $\mathrm{Si}$, en vertu des critères publics, les individus sont identifiés les uns aux autres en raison de leurs qualités objectives inhérentes, les critères perspectivaux d'identification présupposent le point de vue d'un certain sujet effectuant cette identification. Prenons l'exemple de l'identification qu'accomplit, dans le champ visuel, un sujet quelconque nommé Jean. Conformément à l'identification perspectivale, les corps qui occupent la même place dans le champ visuel de Jean peuvent être considérés comme identiques indépendamment des représentations que Jean se fait de leur essence. L'identification perspectivale nous permet de parler de notre environnement « du point de vue subjectif», sans aucune interprétation publique. L'identification publique d'un objet signifie, au contraire, sa localisation dans le domaine de la connaissance intersubjective; autrement dit, il faut donner une compréhension de l'objet en guise de réponse à la question "qu'est-ce que c'est?». Si l'on utilise une comparaison avec le théâtre antique, on peut constater que l'interprétation publique a lieu par rapport à l'acteur lorsqu'il a enlevé le masque et s'est libéré de sa persona perspectivale, c'est-à-dire de la perspective de son identification qui était déterminée par son masque.

Dans ses premières œuvres, désormais classiques, portant sur l'interprétation performative du cogito cartésien, Hintikka a affirmé que «le Cogito peut seulement démontrer l'existence d'une entité perspectivement individuée, non d'une entité publiquement identifiée ${ }^{24}$.

24. J. Hintikka, "Cogito, ergo quis est?», Revue de métaphysique et de morale, t. 105, $\mathrm{n}^{\circ} 1,2000$, p. 20. 
Il a également souligné que

le Cogito cartésien ne saurait guère corroborer quelque inférence que ce soit pour l'existence d'un objet public et a fortiori d'une substance. Pour autant que la Res dans la formule de Descartes Sum res cogitans est censée être une Res publica, cette conclusion prétendue ne s'ensuit justement pas du Cogito ${ }^{25}$.

Cependant, dans son étude: "René pense, donc Descartes existe», Hintikka a reconnu la faiblesse de sa position précédente qui est insuffisante pour justifier le passage du cogito à l'existence du «je » comme chose pensante. Il estime en effet désormais que la chose, même pensante, doit être soumise à l'identification publique. Il semble étrange que là où le premier «je» dans l'expression cartésienne «je pense, donc je suis» est identifié de façon perspectivale, le second «je», celui qui apparaît après "donc», soit déjà une chose, une res cogitans. Cela signifie-t-il qu'il nous faille condamner le cogito en raison de cette alchimie logique, tout comme il faudrait condamner Descartes en raison de la création de la "pierre philosophale», celle de l'implication performative permettant de changer les vils métaux des essences subjectivement identifiées en or de l'objectivité?

Selon Hintikka, il est possible de surmonter la dichotomie dangereuse des «je cartésiens " grâce à la métaphore du spectacle théâtral, où l'acteur existe de deux façons distinctes. À la suite de Hintikka, nous supposons que le premier «je» («je pense») est prononcé par Descartes comme l'acteur qui joue le rôle du sceptique radical, et ce « je » nous l'appellerons René. Que le doute hyperbolique plonge Descartes dans un certain état «simulé», cela est confirmé par le fait que son objectif essentiel n'est pas la preuve mais la réfutation du scepticisme ${ }^{26}$. Descartes interprète donc le rôle du sceptique René, mis en scène par lui-même dans un spectacle «contre-factuel» et dont le "cadre narratif» est donné par le doute radical, celui-ci déterminant la perspective d'«identification narrative» de son personnage René. C'est par conséquent dans le rôle de René que Descartes effectue l'acte mental.

Cependant, l'existence de René est limitée dans le cadre du spectacle; de même que son existence n'y est stable qu'en raison de l'effort permanent du cogito pour la stabiliser. C'est dans un effort permanent semblable, dans cet état d'«obstination tendue» que demeure toujours le héros du roman

25. J. Hintikka, «Cogito, ergo quis est?», 2000, p. 22.

26. Sur la «technique dramaturgique» du cogito, voir par exemple: R. Champigny, «The Theatrical Aspect of the Cogito ", The Review of Metaphysics, vol. 12, nº 3, 1959, p. 370-377; A. O. Rorty, "The Structure of Descartes' Meditations", in Essays on Descartes' Meditations, A. O. Rorty (dir.), Berkeley, University of California Press, 1986, p. 1-2; E. T. Flood, «Descartes's Comedy of Error », MLN, vol. 102, nº 4, 1987, p. 847-866; F. Hallyn, Descartes. Dissimulation et ironie, Genève, Droz, 2006. 
d'Italo Calvino Le chevalier inexistant. Agilulfe ne dort ni le jour ni la nuit, de peur qu'il ne se perde pour toujours s'il s'endort ne serait-ce qu'un instant. En n'existant, selon la volonté de Calvino, que dans les conditions de son inexistence présupposée, Agilulfe est constamment obligé de faire de grands efforts pour accomplir son devoir de chevalier et ne pas disparaître pour toujours ${ }^{27}$.

Au reste, peu importe la façon dont René, sceptique inexistant, accomplit son devoir, en justifiant par la force du cogito son existence à titre de personnage de la "pièce absurde» mise en scène par Descartes. La difficulté principale vient du fait que, pour passer $\mathrm{du}$ «je» du personnage au «je» de l'acteur, le «je» de René qui effectue l'acte mental pendant le spectacle doit devenir le «je» de Descartes qui existe hors de ce spectacle; en d'autres termes, l'acteur doit enlever le masque et rentrer à la maison, c'est-à-dire au fond revenir dans le monde réel. À première vue, un tel passage n'est pas du tout possible parce que tout ce qui se passe avec le personnage n'influence pas l'identification de l'acteur. Quel que soit le nombre des actes mentaux héroïques que René effectue avec enthousiasme, cela n’influence en rien le statut existentiel de l'acteur qui a joué son rôle. Comme le remarque JeanPaul Sartre, «l'acteur est happé, inspiré tout entier par l'irréel. Ce n'est pas le personnage qui se réalise dans l'acteur, c'est l'acteur qui s'irréalise dans son personnage $»^{28}$.

L'acteur qui joue Hamlet pleure réellement, mais ses larmes sont irréelles. Dès lors, on voit non seulement les larmes mais aussi les actes de discours simulés de l'acteur. Il est certain, remarque Hintikka ${ }^{29}$, qu'on peut imaginer une telle situation quand Clint Eastwood, jouant le rôle de l'inspecteur Harry, dit: «Même Clint Eastwood ne pourrait pas garder son air impassible dans une situation semblable». Cette déclaration, faite par l'inspecteur Harry à la première personne, se rapporte à Clint Eastwood qui est un homme réel et peut être vérifiée par l'étude des faits réels de sa vie. Cependant, le jeu par Eastwood du rôle de l'inspecteur Harry n'est qu'un fait accidentel, c'est-à-dire qu'il n'est pas une condition conceptuelle pour réaliser cette déclaration mais sert juste de moyen pour le metteur en scène. Le même moyen est utilisé dans le film policier Le retour de Danny Ocean, où Julia Roberts joue le rôle d'une femme jouant le rôle de Julia Roberts. Cette situation diffère de la performance «tautologique» de Bruce Willis dans ce même film où il joue son propre rôle, lequel convainc le personnage

27. I. Calvino, Le chevalier inexistant, M. Javion (trad.), Paris, Seuil, 1962.

28. J.-P. Sartre, L'imaginaire. Psychologie phénoménologique de l'imagination, Paris, Gallimard (Folio/Essais; 47), 2005, p. 368.

29. Voir à ce sujet l'article de J. Hintikka dans la présente revue. 
de Julia Roberts de l'usurpation d'identité de Julia Roberts. Cependant, comme le souligne Hintikka, le fait que René joue Descartes dans la pièce mise en scène par Descartes représente une des règles institutionnelles de la pièce qui détermine les conditions de l'existence de René. De la même façon, si Descartes affirmait «je suis René Descartes» dans le monde réel, l'affirmation serait conçue par Hintikka comme une vérité conceptuelle de la langue. Il considère comme principe de la compétence linguistique de chaque porteur de la langue la connaissance du fait que le «je» (à la différence du nom propre, par exemple) ne se rapporte pas à l' « incarnation perspectivale» de chaque moi, mais à chaque moi en tant que locuteur publiquement identifié. L'affirmation «je pense» du personnage (René, qui est ainsi perspectivalement identifié) est donc, selon Hintikka, une raison suffisante pour l'affirmation «je suis» par l'auteur (Descartes, qui est à son tour publiquement identifié).

Il est totalement justifié, du point de vue de la logique et de la grammaire, que le «je» ne puisse pas commettre d'erreur sur son identité bien qu'il puisse en commettre en utilisant son nom propre. L'affirmation «je pense, donc Cartesius est» s'énoncerait comme une plaisanterie logique même dans le cas où elle serait prononcée par Descartes. Comme le remarque Hintikka, «il m'est arrivé de voir une bande dessinée française dont tout le sel visait le ridicule du pseudo-Cogito "Je pense, donc Cartesius existe". Ce qui est particulièrement frappant ici, c'est que le ridicule persiste même si je place ces mots dans la propre bouche de Descartes ${ }^{30}$.

Selon Émile Benveniste, le «je» appartient à la réalité unique qui est une réalité du discours. Il écrit: «L'énonciation “je jure” est l'acte même qui m'engage, non la description de l'acte que j'accomplis $»^{31}$.

Il a souligné également que l'énoncé de ce type a «une propriété singulière, celle d'être sui-référentiel, de référer à une réalité qu'il constitue lui-même ${ }^{32}$.

Les données de la linguistique comparative et historique sont une preuve indirecte de la liaison stable entre le pronom je et le locuteur. Dans presque toutes les langues connues, les pronoms de la première personne du singulier proviennent de particules démonstratives appartenant au domaine du locuteur. On peut dire la même chose d'une langue du nord de la Sibérie, le nénéen, dans laquelle tous les autres pronoms personnels ne viennent pas de particules démonstratives mais du substantif pyd (пыд), «соrps».

30. J. Hintikka, «Cogito, ergo quis est?», 2000, p. 21.

31. É. Benveniste, Problèmes de linguistique générale, Paris, Gallimard (Bibliothèque des sciences humaines), 1966, vol. 1, p. 265

32. Ibid., p. 273-274. 
Par exemple: le nénéen dit à partir du substantif pyd (пыд): «corps»; pydar (пыдар): «tu»; pydara (пыдара): «vous»; pyda (пыда): «il»; pydo (пыдо) : «ils»33.

Autrement dit, même si le locuteur du nénéen accepte le fait que «elle», «il», «ils» sont des corps, il accomplit une identification du «je» «de l'intérieur», et cette identification n'est pas déterminée par sa propre identité corporelle, ce qui correspond précisément à l'esprit de l'argument cartésien.

Cependant, le développement des technologies contemporaines pose de nouveaux problèmes qui ne se sont pas posés à nos ancêtres, les créateurs de la langue. L'un de ces problèmes a été engendré par l'invention du répondeur téléphonique. Si on écoute au téléphone la phrase: «Je ne suis pas à la maison, laissez votre message après le signal», nous ne supposons pas qu'on entre en communication avec le chevalier inexistant Agilulfe qui effectue un acte de discours là où il n'existe pas. On ne soupçonne pas non plus notre interlocuteur de mensonge comme s'il imitait son propre répondeur en faisant des grimaces, même s'il est vrai qu'il a commis un mensonge quand il a enregistré cette phrase à la maison. Cette situation est reproduite dans le film Sam, je suis Sam où le héros, qui a l'âge mental d'un enfant et fait preuve pour cela d'une honnêteté absolue, refuse d'enregistrer sur le dictaphone la phrase disant qu'il ne se trouve pas à la maison quand il y est. On peut supposer que Sam refuserait de jouer le rôle d'Hamlet, puisqu'il serait obligé d'accomplir des actes de discours mensongers. D'où la question suivante: la performance sincère de l'acte de discours est-elle une condition nécessaire à l'appartenance $\mathrm{du}$ «je» au locuteur? Ni le discours du répondeur, ni le discours de l'acteur prononçant le monologue de Hamlet à la première personne, ni la réplique de René «je pense» ne satisfont évidemment à cette condition.

La nouvelle interprétation faite par Hintikka laisse encore une question ouverte. La perspective d'identification de René est déterminée par l'auteur de la pièce, c'est-à-dire par Descartes. L'auteur de la pièce doit donc être identifié au préalable; et puisqu'il est une source de la perspective, cette identification ne peut pas être perspectivale à son tour. Dans le cas contraire, la pluralité des perspectives signifierait la pluralité des auteurs.

Vincent Carraud note:

Concernant Descartes, le constat s'impose: l'ego est, dans les Meditationes, le sujet grammatical (le je du locuteur), et ne devient jamais l'objet de la pensée d'autrui, $\mathrm{ni}$, a fortiori, de son regard ou de son amour: aucun déplacement de point de vue, aucun décentrement ne viennent rompre la monologie égologique ${ }^{34}$.

33. К. Е. Майтинская, Местоимения в языках разных систем, Moscou, 2009, р. 206.

34. V. Carraud, L'invention du moi, Paris, PUF, 2010, p. 44. 
D'autre part, le traitement de l'identification perspectivale du je comme identification à partir de ma propre perspective (l'identification du «je» dans la perspective $\mathrm{du}$ «je») conduit à une régression à l'infini propre à l' «égologie cognitive». Comme le remarque Vincent Descombes, les partisans de cette égologie considèrent naturel le passage de la tautologie «je suis moi» à la thèse métaphysique « je suis quelqu'un qui est moi». Ils croient que le discours à la première personne garantit la "prise cognitive» de soi-même par le sujet ${ }^{35}$.

Malheureusement, dans la tradition analytique, la perspective de la première personne est souvent liée à la réflexion propositionnelle. Comme l'écrit par exemple Lynne Rudder Baker,

on a la perspective de la première personne si et seulement si on a la capacité de se concevoir comme étant soi-même, cette capacité étant signalée par la capacité linguistique d'attribuer la (et de faire) référence de la première personne à soi-même ${ }^{36}$.

Une telle position risque d'amener au «représentativisme d'ordre supérieur» décrit par le phénoménologue Dan Zahavi de la façon suivante:

Les théories de la représentation de l'ordre supérieur prétendent qu'un certain état mental, pour se manifester phénoménalement (et ne pas rester simplement inconscient), doit atteindre son objectivation par une pensée ou perception consécutive de second ordre ${ }^{37}$.

L'état mental se transforme donc en une propriété relationnelle: afin d'être pris en compte, l'état mental doit être accompagné par la représentation correspondante d'ordre supérieur, c'est-à-dire par la pensée de second ordre sur cet état ou par sa perception de second ordre. Cependant, le cogito pré-réflexif est autosuffisant et ne se déduit pas de l'acte secondaire de la réflexion ${ }^{38}$.

35. V. Descombes, Le complément de sujet: enquête sur le fait d'agir soi-même, Paris, Gallimard (NRF essais), 2004.

36. L. R. Baker, Persons and Bodies: A Constitution View, Cambridge, Cambridge University Press, 2000, p. 68: "One has a first-person perspective if and only if one has the ability to conceive of oneself as oneself, where this ability is signaled by the linguistic ability to attribute (as well as to make) first-person reference to oneself».

37. D. Zahavi, «First-Person Thoughts and Embodied Self-Awareness: Some Reflections on the Relation between Recent Analytical Philosophy and Phenomenology", Phenomenology and the Cognitive Sciences, vol. 1, $\mathrm{n}^{\circ}$ 1, 2002, p. 15-16: "The higher-order representation theories claim that a certain mental state in order to manifest itself phenomenally (and not merely remain unconscious) must await its objectification by a subsequent second-order thought or perception".

38. Selon Husserl, les compréhensions de second ordre ne commencent pas à s'établir sur la compréhension du soi. Jean-Paul Sartre avance que la conscience de soi n'est pas couplée avec la conscience réfléchie. 
L'identification du je, sans être perspectivale, ne peut pas non plus être examinée comme une identification publique de type «qu'est-ce que c'est?». Vincent Carraud écrit:

chez Descartes, le référé du pronom n'est pas une personne particulière, empirique, René Descartes lui-même, mais ce ou celui qui a résisté au doute et qui, ayant résisté au doute, est, et n'est pour l'instant que cette résistance même, résidu pur et solide du moi personnel dont le doute a eu raison de toutes les particularités et de toutes les déterminations ${ }^{39}$.

Il s'agit non pas de la personne de Descartes identifiée publiquement, mais de "ce qui en reste après le travail du doute ${ }^{40}$. Comme Carraud conclut aphoristiquement: «le moi, ce n'est pas moi ${ }^{41}$.

On se trouve ainsi devant le dilemme suivant: l'identification du «moi» ne peut être ni l'identification publique de type «qu'est-ce que c'est? ", ni l'identification perspectivale dans la perspective de quelqu'un, y compris dans ma propre perspective réflexive. L'affirmation de l'identification performative de type «qui est-ce?», permet, me semble-t-il, de surmonter ce dilemme. C'est, en substance, de ce type d'identification que Ludwig Wittgenstein a parlé. On connaît bien les aphorismes du Tractatus logicophilosophicus dirigés contre l'idée du sujet pensant comme une partie du monde. Le plus connu de ces aphorismes est le paragraphe 5.633: «Où, dans le monde, un sujet métaphysique peut-il être discerné ?» demande Wittgenstein qui répond lui-même: "Tu réponds qu'il en est ici tout à fait comme de l'œil et du champ visuel. Mais l'œil, en réalité, tu ne le vois pas. Et rien dans le champ visuel ne permet de conclure qu'il est vu par un œil» ${ }^{42}$.

Dans ses réflexions tardives sur la nature du champ visuel, Wittgenstein fixe l'attention sur son caractère phénoménologique. Dans les Recherches philosophiques, il montre ainsi l'inefficacité de l'analogie entre le champ phénoménal et l'espace physique, en nous proposant d'imaginer un fauteuil conscient de soi:

Le fauteuil pense par-devers soi que...

Où pense-t-il ? Dans l'une de ses parties? Ou à l'extérieur de son corps, dans l'air qu'il y a autour? Ou nulle part? Mais quelle est la différence entre la

39. V. Carraud, L'invention du moi, p. 48.

40. Ibid., p. 49.

41. Ibid., p. 63.

42. L. Wittgenstein, Tractatus logico-philosophicus, Paris, Gallimard (Bibliothèque de philosophie), 1993, p. 94. 
parole intérieure de ce fauteuil et celle d'un autre fauteuil qui se trouve à côté de lui ? - Qu'en est-il donc de l'homme: Où se parle-t-il à lui-même? Comment se fait-il que cette question semble vide de sens ${ }^{43}$ ?

Chez Wittgenstein le champ visuel n'est pas identique à l'espace physique; il est plus proche du champ phénoménal des phénoménologues. Le champ phénoménal ne se trouve pas «à l'intérieur du sujet». Comme le dit Maurice Merleau-Ponty,

ce champ phénoménal n'est pas un «monde intérieur», le "phénomène» n'est pas un «état de conscience» ou un «fait psychique», l'expérience des phénomènes n'est pas une introspection ou une intuition au sens de Bergson ${ }^{44}$.

L’attitude phénoménologique ne nous plonge pas dans les «ténèbres du monde intérieur». Selon Merleau-Ponty,

c'est la notion même d'immédiat qui se trouve transformée: est désormais immédiat non plus l'impression, l'objet qui ne fait qu'un avec le sujet, mais le sens, la structure, l'arrangement spontané des parties ${ }^{45}$.

Ainsi, l'évidence de l'immédiateté n'est pas propre à ce que Hume décrit quand il note: «Pour moi, quand je pénètre le plus intimement dans ce que j'appelle moi-même, je tombe toujours sur une perception particulière ou sur une autre, de chaleur ou de froid, de lumière ou d'ombre, d'amour ou de haine, de douleur ou de plaisir " ${ }^{46}$, mais concerne l'ordre sémantique des perceptions en général. Cet ordre est déterminé par la perspective subjective. Par suite, l'hypothèse d'une langue sans sujet est une illusion.

Comment cette perspective apparait-elle à son tour? Selon Wittgenstein, l'énonciation de la première personne n'est pas une énonciation ordinaire du monde mais constitue une action, un évènement dans le monde. La différence entre les propositions «j'ai mal» et «il a mal» correspond plutôt à la différence entre le gémissement et l'énonciation disant que quelqu'un gémit ${ }^{47}$.

43. L. Wittgenstein, Recherches philosophiques, F. Dastur (trad.), E. Rigal (éd.), Paris, Gallimard, 2005, \$361, p. 167.

44. M. Merleau-Ponty, Phénoménologie de la perception, $2^{\mathrm{e}}$ éd., Paris, Gallimard, 1945, p. 69-70.

45. Ibid., p. 70

46. D. Hume, Traité de la nature humaine, in L'entendement, liv. I, part. IV, section VI, P. Baranger, P. Saltel (trad.), Paris, Flammarion (GF; 701), 1995, p. 344.

47. L. Wittgenstein, Le "Cahier bleu" et le "Cahier brun", Paris, Gallimard (Bibliothèque de philosophie), 1996. 
L'extension des principes d'identification performative au «moi» cartésien peut sans doute être critiquée dans le cadre de la conception $\mathrm{du}$ «théâtre interne», que l'on appelle souvent le «théâtre cartésien » ${ }^{48}$. Cela dit, la métaphore du "théâtre interne», qui présuppose l'existence d'un espace mental privé, autrement dit d'une intériorité mentale, est en fait bien présentée chez Hume plutôt que chez Descartes, dans le cadre du contexte polémique avec ce dernier. Hume écrit: "L'esprit est une sorte de théâtre, où des perceptions diverses font successivement leur entrée, passent, repassent, s'esquivent et se mêlent en une variété infinie de positions et de situations ${ }^{49}$.

Dans la "Méditation troisième ", Descartes utilise à son tour les métaphores «internalistes» de l'image et du tableau. Il remarque que «[...] les idées sont en moy comme des tableaux, ou des images [imagines] $\aleph^{50}$. Cependant, la compréhension cartésienne de l'intériorité est distincte de l'introspectionnisme psychologique classique ${ }^{51}$. Dans les «Réponses» aux «Objections» de Gassendi, Descartes souligne:

[...] ce n'est point l'œil qui se voit lui-même ni le miroir, mais bien l'esprit, lequel seul connaît et le miroir, et l'œil, et soi-même ${ }^{52}$.

Comme l'écrit Descartes dans la «Méditation seconde»,

Mais qu'est-ce que donc que je suis? Une chose qui pense. Qu'est-ce qu'une chose qui pense? C'est-à-dire une chose qui doute, qui conçoit, qui affirme, qui nie, qui veut, qui ne veut pas, qui imagine aussi, et qui sent ${ }^{53}$.

D'autre part, le caractère performatif de l'identification du «moi» engendre encore une question difficile: la pluralité des actions du «je»

48. Par exemple, H. Putnam parle de la «conception propre à Descartes du mental comme une sorte de théâtre intérieur» (H. Putnam, The Threefold Cord: Mind, Body, and World, New York, Columbia University Press, 1999, p. 101); D. Dennett attribue à Descartes la conception d'un «théâtre central» (D. C. Dennett, Consciousness Explained, Boston, Little, Brown and Co., 1991). Voir aussi P. Gillot, «La question de l'intériorité mentale à l'âge classique", Revue de synthèse, vol. 131, 2010, p. 7-20.

49. D. Hume, Traité de la nature humaine, p. 344.

50. AT IX, 33; AT VII, 42 pour le texte latin.

51. Voir par exemple G. Canguilhem, "Qu'est-ce que la psychologie?», Revue de métaphysique et de morale, $\mathrm{n}^{\circ} 1,1958$, p. 12-25; rééd. dans G. Canguilhem, Études d'histoire et de philosophie des sciences, Paris, Vrin (Problèmes et controverses), 1994, p. 365-381.

52. Traduction française, Alquié II, 810; original latin, AT VII, 367 : «non esse oculum qui speculum videt magis quam seipsum, sed mentem quae sola, et speculum, et oculum, et seipsam quoque, agnoscit».

53. AT IX-1, 22. 
conduit-elle à sa dispersion en «je» pluriels, où chacun s'identifie-t-il à travers ses propres actions?

Comme le remarque Jean-Luc Marion, le modèle du performatif

ne cherche jamais à établir une existence nue, pure et universelle - ego sum, ego existo -, mais seulement qu'une énonciation particulière produit effectivement un résultat; ce résultat (inculpation, vœux, interdiction, promesse, etc.) implique certes une existence, mais toujours déterminée: celle qui correspond à la qualification de l'énonciateur ou au sens de l'énoncé; l'existence ainsi performée admet des conditions, donc aussi des limites ${ }^{54}$.

Selon Descartes, c'est Dieu qui rend le «moi » constant. Descartes demande ainsi :

[...] comment seroit-il possible que je peusse connoistre que je doute et que je désire [...] si je n'avois en moy aucune idée d'un estre plus parfait que le mien, par la comparaison duquel je connoistrois les defauts de ma nature ${ }^{55}$ ?

Dans ses «Réponses aux secondes objections», juste avant ses remarques sur le caractère non-syllogistique du cogito, il souligne:

[...] j'ay dit que nous ne pouvons rien sçavoir certainement, si nous ne connoissons premierement que Dieu existe, j'ay dit, en termes exprez que je ne parlois que de la science de ces conclusions, dont la mémoire nous peut revenir en l'esprit, lorsque nous ne pensons plus aux raisons d'où nous les avons tirées ${ }^{56}$.

Par conséquent, l'identification cartésienne de type « qui est-ce?» s'effectue dans la perspective unique et exacte déterminée par le véritable auteur. Cette perspective représente en même temps la conscience de la «marque» de cette autorité; autrement dit, il ne s'agit pas d'un «souci de soi» réflexif et égotique mais de la transcendance. Selon Descartes,

comme l'idée de moy-mesme, elle [l'idée de Dieu] est née et produite avec moy dés lors que j'ay esté creé. Et certes on ne doit pas trouver estrange que Dieu, en me créant, ait mis en moy cette idée pour estre comme la marque de l'ouvrier emprainte sur son ouvrage; et il n'est pas aussi necessaire que cette marque soit quelque chose de different de ce mesme ouvrage. Mais de cela seul que Dieu m'a creé, il est fort croyable qu'il m'a en quelque façon produit à son image et semblance, et que je conçoy cette ressemblance (dans

54. J.-L. Marion, Sur la théologie blanche de Descartes, Paris, PUF (Quadrige. Essais, débats), 2009, p. 382.

55. AT IX-1, 36; VII, 42 pour le texte latin.

56. AT IX-1, 110. 
laquelle l'idée de Dieu se trouve contenuë) par la mesme faculté par laquelle je me conçoy moy-mesme ${ }^{57}$.

La «marque de l'auteur» sur ses ouvrages se retrouve dans le degré d'authenticité du cogito. Ce n'est donc pas seulement René qui est un acteur dans le spectacle mis en scène par Descartes: c'est Descartes lui-même dont l'existence est fondée sur la véracité divine. Il est donc décisif de prendre en considération le fait que l'auteur de la pièce dans laquelle joue Descartes n'est pas trompeur.

Elena Dragalina-Chernaya Université d'État, École des hautes études en sciences économiques, Moscou 